\title{
Risk Factors of Atrial Fibrillation in Patients with Heart Failure
}

\author{
Alam Sarfraz ${ }^{1}$, Parveen Akhtar ${ }^{1}$, Syed N. Hassan Rizvi ${ }^{1}$, Musa Karim ${ }^{2}$ \\ 1. Cardiology, National Institute of Cardiovascular Diseases, Karachi, PAK 2. Miscellaneous, National Institute of \\ Cardiovascular Diseases, Karachi, PAK
}

Corresponding author: Musa Karim, mkarim.nicvd@gmail.com

\section{Abstract \\ Introduction}

Coexistence of atrial fibrillation (AF) in patients with heart failure (HF) is a common phenomenon associated with poor prognosis. Therefore, this study was designed with an aim to estimate the different risk factors of atrial fibrillation (AF) in patients with HF.

\section{Methods}

In this study, patients of either gender, 18 to 80 years of age, and with echocardiographic confirmation of HF presenting at the adult cardiology department of the National Institute of Cardiovascular Diseases (NICVD), Karachi, Pakistan were consecutively included. Patients diagnosed with chronic obstructive airway diseases, pneumonia, or pericarditis, and patients diagnosed with existing AF were excluded from the study. Data regarding demographic and clinical risk factors of AF were obtained using a structural proforma.

\section{Results}

Out of 150 patients, $59.3 \%$ (89) were females, and the mean age was $50 \pm 16$ years. A majority of the patients, 55.3\% (83), had a history of rheumatic heart diseases (RHD) and 22.7 (34) percent had a history of transient ischemic attack (TIA) or cerebrovascular accident (CVA). On echocardiography, 28.0\% (42) of the patients had right ventricular (RV) dysfunction, and the clot was seen in 28.0\% (42) of the patients. Mitral stenosis (MS) and mitral regurgitation (MR) were observed in $34.5 \%$ (61) and $29.3 \%$ (52) of the patients, respectively.

\section{Conclusion}

We observed that the adult population with HF tends to have multiple risk factors of AF. More coordinated efforts are needed by the healthcare professionals to understand and manage these coupled conditions.

Received 12/12/2018

Review began 12/16/2018 Review ended 12/20/2018 Published 12/24/2018

\section{(๑) Copyright 2018}

Sarfraz et al. This is an open access article distributed under the terms of the Creative Commons Attribution License CC-BY 3.0., which permits unrestricted use, distribution, and reproduction in any medium, provided the original author and source are credited.
Categories: Cardiology

Keywords: atrial fibrillation, risk factors, heart failure

\section{Introduction}

Cardiovascular diseases (CVD) account for nearly $10 \%$ of the worldwide burden of diseases, measured by a combination of death and disability, and this is expected to rise to almost $15 \%$ by 2020 [1]. Heart failure (HF) is the most commonly observed manifestation of the acute coronary syndrome (ACS) and is related to increased adverse outcomes [2-3]. HF accounts for more than a 100 billion dollars of a direct and indirect global economic burden. Middle- and low-income countries bear the major share of indirect cost [4]. Coexistence of atrial fibrillation (AF) and HF is a common phenomenon, with both worsening the course of one another. The presence of both the conditions in a patient is associated with poor prognosis [5]. A complex interplay exists between $\mathrm{HF}$ and $\mathrm{AF}$, and the development of AF is influenced by HF, while the stimulation of HF is influenced by AF. HF with both reduced and preserved ejection fraction (EF) is worsened by the preexisting AF. The individual clinical factors known to increase a patient's susceptibility include advanced age, diabetes mellitus (DM), and congestive heart failure (CHF) [6].

The prevalence of AF in the European population is around 1\% to $2 \%$; however, it has been increasingly observed in adultery population [7]. Depending on the age of the patients and the type of HF, the prevalence rate of $\mathrm{AF}$ varies from $13 \%$ to $41 \%$ [4,8]. A study conducted on 10,701 patients with $\mathrm{HF}$, admitted to the hospital in 24 countries in Europe, demonstrated that only $9 \%$ had new-onset AF, while $34 \%$ had preexisting $\mathrm{AF}[9]$.

Clinical risk factors for the development of $\mathrm{AF}$ in the general population have been characterized by different risk factors like age, race, body weight, and height, and blood pressure, antihypertensive medication, diabetes, and a history of myocardial infarction (MI), and CVDs [10-12]. In addition to structural 
heart diseases, a number of other factors may contribute to the development of AF including inflammation and autonomic. The prognostic impact of AF in patients with HF is well established. A number of studies have reported a deleterious effect on outcomes such as increased risk of mortality, stroke, and morbidity [13].

The frequency and risk factors of AF in the Pakistani population are not known. Therefore, this study was designed with the rationale to estimate the different risk factors of AF in patients with HF.

\section{Materials And Methods}

This cross-sectional study was performed at the adult cardiology department of the National Institute of Cardiovascular Diseases (NICVD), Karachi, Pakistan from February 2017 to July 2017. This study was approved by the ethical review committee (ERC) of the institution (ERC approval number: ERC-07/2017). In this study, patients of either gender above 18 years of age with echocardiographic confirmation of HF were consecutively included. Informed consent regarding participation in this study was obtained from all the enrolled patients. Exclusion criteria for the study were patients below 18 years or above 80 years of age, patients diagnosed with chronic obstructive airway diseases, pneumonia, or pericarditis, and patients diagnosed with existing AF. AF was diagnosed based on electrocardiographic (ECG) characteristics including irregular R-R intervals (when atrioventricular conduction is present), an absence of distinct repeating $\mathrm{P}$ waves, and irregular atrial activity. The risk factors of $\mathrm{AF}$ were categorized as demographic characteristics (advanced age and obesity), clinical manifestations (blood pressure, heart rate, shortness of breath, angina, dizziness, palpitation, and orthopnea), clinical history (hypertension (HTN), dyslipidemia, DM, and history of transient ischemic attack [TIA], ischemic heart diseases [IHD], cerebrovascular accident [CVA], MI, and rheumatic heart diseases [RHD]), ECG changes, and echocardiography findings (the presence of clot, valvular lesions, right ventricular [RV] dysfunction, left atrial size, and pulmonary arterial pressure [PAP]). ECG and echocardiographic findings were analyzed independently by three cardiologists and the agreement of any two regarding a certain finding was considered as the final finding. All the collected information was recorded on a pre-defined structural proforma.

After a thorough literature review, no data were available regarding the risk factors of AF in patients with $\mathrm{HF}$ for the Pakistani population; therefore, the sample size for the study was calculated at $95 \%$ confidence level, with $50 \%$ prevalence guess, and $8 \%$ margin of error, and based on these assumptions, the minimum achievable sample size for the study was 151 .

Collected data were entered and evaluated for data entry and collection errors, and data analysis was carried out using IBM SPSS Statistics for Windows, Version 21.0. (IBM Corp., Armonk, NY, US). No imputation was performed for the variables with missing data; rather such cases were excluded list-wise from the analysis. Under the assumption of normality of the distribution, quantitative (continuous) information was summarized by calculating the mean \pm standard deviation $(\mathrm{SD})$, while for the categorical variables, the frequency and percentage were computed.

\section{Results}

A total of 150 patients were included in this study: $59.3 \%$ (89) were females, and the mean age was $50 \pm 16$ years. A majority, $94.0 \%$ (141), of the patients were married and $19.3 \%$ (29) were above 65 years of age. A majority of the patients, $55.3 \%$ (83), had a history of RHDs, $22.7 \%$ (34) had a history of TIAs or CVAs. Majority of the patients, $89.3 \%$ (134), presented with SOB class IV. Demographic characteristics, clinical manifestations, and clinical history of the patients are presented in Table 1. 


\section{Cureus}

Characteristics

Percentage ( $n$ )

Gender distribution

Female

$59.3(89)$

Male

Marital Status

Married

Unmarried

Age distribution

18 to 40 years

30.7 (46)

41 to 65 years

$50.0(75)$

More than 65 years

$19.3(29)$

Clinical history

Hypertension

42.7 (64)

Diabetes

6.7 (10)

Smoker

8.0 (12)

Ischemic heart disease (IHD)

2.7 (4)

Myocardial infarction (MI)

$5.3(8)$

Rheumatic heart diseases (RHD)

$55.3(83)$

Transient ischemic attack (TIA) / Cerebrovascular accident (CVA)

Clinical manifestation

Systolic blood pressure (SBP)

$115.5 \pm 21.5 \mathrm{mmHg}$

Pulse rate

$123.6 \pm 29.6 \mathrm{bpm}$

Serum creatinine

$1.1 \pm 0.6 \mathrm{mg} / \mathrm{dL}$

Hemoglobin (HB)

$11.7 \pm 1.9 \mathrm{gm} / \mathrm{dL}$

Shortness of breath (SOB - IV)

89.3 (134)

Angina

$5.3(8)$

Dizziness

$35.3(53)$

Palpitation

78.7 (118)

Orthopnea

46.0 (69)

TABLE 1: Demographic characteristics, clinical manifestations, and clinical history

Radiological cardiomegaly was observed in $66.0 \%$ (99) of the patients, left ventricular hypertrophy (LVH) on ECG was observed for a majority, $40.0 \%$ (60), of the patients, and 31.3\% (47) of the ECGs were normal. In $28.0 \%$ (42) of the patients, clots were on observed on ECG, and RV dysfunction was observed in $28.0 \%$ (42) of the patients. The major valvular lesions were mitral stenosis (MS) and mitral regurgitation (MR), which were observed in $34.5 \%$ (61) and $29.3 \%$ (52) of the patients, respectively. ECG changes and echocardiography findings are presented in Table 2 . 


\section{Cureus}

\begin{tabular}{|l|l|}
\hline Characteristics & Percentage $(n)$ \\
\hline ECG Changes & \\
\hline Ischemic Changes & $22.0(33)$ \\
Left ventricular hypertrophy (LVH) & $40.0(60)$ \\
\hline Left bundle branch block (LBBB)/Right bundle branch block (RBBB) & $6.7(10)$ \\
\hline Normal & $31.3(47)$ \\
\hline Echocardiography findings & \\
\hline Right ventricular (RV) dysfunction & $29.3(44)$ \\
\hline Presence of Clot & $28.0(42)$ \\
\hline Valvular Lesion & \\
\hline Mitral stenosis (MS) & $34.5(61)$ \\
\hline Tricuspid regurgitation (TR) & $19.8(35)$ \\
\hline Mitral regurgitation (MR) & $29.3(52)$ \\
\hline Aortic regurgitation (AR) & $1.7(3)$ \\
\hline Aortic stenosis (AS) & $1.2(2)$ \\
\hline Other Lesions & $13.5(24)$ \\
\hline Left atrial (LA) size & $46.7 \pm 14.0 \mathrm{~mm}$ \\
\hline Pulmonary arterial pressure (PAP) & $39.4 \pm 13.8 \mathrm{mmHg}$ \\
\hline
\end{tabular}

\section{TABLE 2: ECG changes and echocardiography findings}

ECG: electrocardiography

\section{Discussion}

The study of risk factors of AF in adult individuals is important in the current era of preventive cardiology. This study was conducted with the aim of estimating the burden of the risk factors of AF in patients presenting with HF. Studies conducted in the past have identified various clinical risk factors such as advanced age, body mass index (BMI), HTN, DM, and past history of MI [10-12]. Although it is not well established whether HF induces AF or vice versa; however, the pre-existence of AF in patients with $\mathrm{HF}$ is an independent prognostic marker.

In our study, the mean age of the patients was $50 \pm 16$ years, ranging between 19-75 years, and a majority, $50.0 \%$ (75), of the patients belong to the age groups of 41 to 65 years. Only $19.3 \%$ (29) patients were above 65 years of age. We observed an intense risk profile of AF in our study sample with hypertension in $42.7 \%$, history of RHDs in 55.3\%, and a history of TIA/CVA in $22.7 \%$ of the patients. RV dysfunction was observed in $29.3 \%$, and echocardiographic assessment showed the presence of a clot in $28.0 \%$ of the patients. Among other risk factors, MS was observed in $34.5 \%$ of the patients and MR was found in $29.3 \%$ of the patients. Clinical history of RHDs and HTN are the primary determinants of AF and other ceaseless cardiovascular sicknesses as reported in numerous studies [13-15].

Most of the patients in our study were females (59.3\%) which is comparable to the observations done by other studies [16-17]. A family history of IHDs was found in $4 \%$ of our patients, which is much less than $17.3 \%$ reported in a study conducted in London by Khazanie et al. [16]. A history of MI was present in 5.3\% and $8 \%$ of the patients were smokers. Finally, in our study, we observed that adult population with HF tends to have multiple risk factors for the development of AF. Meticulous counseling and management strategies need to be put forth for this population to minimize the prognostic impact of AF in patients with HF. Even with this information, many questions regarding adult patients with HF still exist, for instance, no data are available regarding follow-up, and the effect of treatment strategies on morbidity and mortality has not been definitively reported for our population in these patient groups. In order to answer these questions, prospective studies with multivariate analysis of risk factors are recommended. First-degree relatives of patients with HF should be educated and screened for the presence of traditional risk factors of AF. 


\section{Conclusions}

We observed that the adult population with HF tends to have multiple risk factors of AF. Therefore, more coordinated efforts are needed by healthcare professionals to understand and manage these coupled conditions.

\section{Additional Information \\ Disclosures}

Human subjects: Consent was obtained by all participants in this study. National Institute of Cardiovascular Diseases, NICVD, Karachi, Pakistan issued approval ERC-07/2017. This study was approved by the ethical review committee (ERC) of the National Institute of Cardiovascular Diseases, NICVD, Karachi, Pakistan (ERC approval number: ERC-07/2017). Animal subjects: All authors have confirmed that this study did not involve animal subjects or tissue. Conflicts of interest: In compliance with the ICMJE uniform disclosure form, all authors declare the following: Payment/services info: All authors have declared that no financial support was received from any organization for the submitted work. Financial relationships: All authors have declared that they have no financial relationships at present or within the previous three years with any organizations that might have an interest in the submitted work. Other relationships: All authors have declared that there are no other relationships or activities that could appear to have influenced the submitted work.

\section{References}

1. Yeates K, Lohfeld L, Sleeth J, Morales F, Rajkotia Y, Ogedegbe O: A global perspective on cardiovascular disease in vulnerable populations. Can J Cardiol. 2015, 31:1081-93. 10.1016/j.cjca.2015.06.035

2. Gheorghiade M, Bonow RO: Chronic heart failure in the United States: a manifestation of coronary artery disease. Circulation. 1998, 97:282-9. 10.1161/01.cir.97.3.282

3. French JK, Armstrong PW, Cohen E, et al.: Cardiogenic shock and heart failure post-percutaneous coronary intervention in ST-elevation myocardial infarction: observations from "Assessment of Pexelizumab in Acute Myocardial Infarction”. Am Heart J. 2011, 162:89-97. 10.1016/j.ahj.2011.04.009

4. Cook C, Cole G, Asaria P, Jabbour R, Francis DP: The annual global economic burden of heart failure . Int J Cardiol. 2014, 171:368-76. 10.1016/j.ijcard.2013.12.028

5. Chamberlain AM, Redfield MM, Alonso A, Weston SA, Roger VL: Atrial fibrillation and mortality in heart failure: a community study. Circ Heart Fail. 2011, 4:740. 10.1161/CIRCHEARTFAILURE.111.962688

6. Liang JJ, Callans DJ: Ablation for atrial fibrillation in heart failure with reduced ejection fraction . Card Fail Rev. 2018, 4:33-7. 10.15420/cfr.2018:3:1

7. Chugh SS, Havmoeller R, Narayanan K, et al.: Worldwide epidemiology of atrial fibrillation: a global burden of disease 2010 study. Circulation. 2014, 129:837-47. 10.1161/CIRCULATIONAHA.113.005119

8. Bui AL, Horwich TB, Fonarow GC: Epidemiology and risk profile of heart failure . Nat Rev Cardiol. 2011, 8:30-41. 10.1038/nrcardio.2010.165

9. Rivero-Ayerza M, Scholte op Reimer W, Lenzen M, et al.: New-onset atrial fibrillation is an independent predictor of in-hospital mortality in hospitalized heart failure patients: results of the EuroHeart Failure Survey. Eur Heart J. 2008, 29:1618-24. 10.1093/eurheartj/ehn217

10. Schnabel RB, Sullivan LM, Levy D, et al.: Development of a risk score for atrial fibrillation (Framingham Heart Study): a community-based cohort study. Lancet. 2009, 373:739-45. 10.1016/S0140-6736(09)60443-8

11. Alonso A, Krijthe BP, Aspelund T, et al.: Simple risk model predicts incidence of atrial fibrillation in a racially and geographically diverse population: the CHARGE-AF consortium. J Am Heart Assoc. 2013, 2:e00102. 10.1161/JAHA.112.000102

12. Chamberlain AM, Agarwal SK, Folsom AR, et al.: A clinical risk score for atrial fibrillation in a biracial prospective cohort (from the Atherosclerosis Risk in Communities [ARIC] study). Am J Cardiol. 2011, 107:85-91. 10.1016/j.amjcard.2010.08.049

13. Murakami N, Tanno M, Kokubu N, et al.: Distinct risk factors of atrial fibrillation in patients with and without coronary artery disease: a cross-sectional analysis of the BOREAS-CAG Registry data. Open Heart. 2017, 4:e000573. 10.1136/openhrt-2016-000573

14. Wolf PA, Abbott RD, Kannel WB: Atrial fibrillation as an independent risk factor for stroke: the Framingham Study. Stroke. 1991, 22:983-8. 10.1161/01.STR.22.8.983

15. Hamaguchi S, Yokoshiki H, Kinugawa S, et al.: Effects of atrial fibrillation on long-term outcomes in patients hospitalized for heart failure in Japan. Circ J. 2009, 73:2084-90. 10.1253/circj.CJ-09-0316

16. Khazanie P, Liang L, Qualls LG, et al.: Outcomes of Medicare beneficiaries with heart failure and atrial fibrillation. JACC Heart Fail. 2014, 2:41-48. 10.1016/j.jchf.2013.11.002

17. Tromp J, Teng TH, Tay WT, et al.: Heart failure with preserved ejection fraction in Asia . Eur J Heart. 2018, [Epub ahead of print]. 10.1002/ejhf.1227 